\title{
Evaluación del tiempo de inducción y la concentración de metanol en la expresión de L-asparaginasa II de Saccharomyces cerevisiae usando Pichia pastoris (Muts)
}

\author{
Omar Pillaca-Pullo, Karin Torres, Marcela Pimenta, Adalberto Pessoa-Jr \& Michele Vitolo \\ Facultad de Ciencias Farmacéuticas - Universidad de São Paulo (Brasil), 0550-8000
}

DOI: https://doi.org/10.33017/RevECIPeru2015.0020/

\begin{abstract}
Resumen
La levadura metilotrófica Pichia pastoris es ampliamente usada como un sistema eucariota para expresar proteínas recombinantes. Más de 500 proteínas recombinantes fueron expresas por P. pastoris con niveles de expresión que alcanzan hasta el $80 \%$ de proteínas totales secretadas y hasta $30 \%$ de proteínas totales de la célula. Existen tres fenotipos de P. pastoris clasificados de acuerdo con su capacidad de metabolizar metanol, el fenotipo MutS crece lentamente en medios con metanol por lo que generalmente se usan bajas concentraciones de metanol y tiempo de inducción prolongados. Por esta razón, el control de las condiciones de cultura como la concentración del inductor y el tiempo de inducción son factores importantes tanto para el crecimiento de la levadura como para producción de la proteína ya que este sistema es controlado por el promotor AOX inducido con metanol. Por otro lado, L-asparaginase (EC. 3.5.1.1) es un importante biofármaco usado en el tratamiento de leucemia linfoblástica aguda (ALL), la enzima comúnmente utilizada en la terapéutica es procedente de bacterias, estas han demostrado buena actividad, pero causan muchas reacciones inmunológicas severas en los pacientes tratados. La búsqueda de L-asparaginasa procedente y expresada en organismos eucariotas se abre como una posibilidad para reducir las reacciones adversas. En este estudio fueron evaluados el tiempo de inducción (24 - 120 horas) y la concentración de inductor $(0.25$, 0.5 y 1.0\%). Los datos mostraron que la condición de mayor expresión de L-asparaginasa II de Saccharomyces cerevisiae después de 48 horas de inducción con 1,0\% de metanol ( 25 U.g-1). Finalmente se recomienda evaluar dicha producción en biorreactor donde se lleve un control adecuado de otras variables importantes como el pH del cultivo y la concentración de oxígeno en el medio.
\end{abstract}

Descriptores: Pichia pastoris, Saccharomyces cerevisiae, L-asparaginase, Metanol. Abstract
The methylotrophic yeast Pichia pastoris is widely used as a eukaryotic system for expressing recombinant
proteins. Over 500 recombinant proteins were expressed in P. pastoris with expression levels reaching up to
$80 \%$ of total secreted proteins and up to $30 \%$ total cell proteins. There are three phenotypes of P. pastoris
classified according to their ability to metabolize methanol, phenotype MutS grows slowly on media containing
methanol at generally low concentrations of methanol and longer induction time are used. Therefore, the
control of culture conditions such as concentration of the inducer and the induction time are important factors
for both yeast growth and for production of the protein since this system is controlled by the AOX promoter
induced with methanol. Furthermore, L-asparaginase (EC. 3.5.1.1) is an important biopharmaceutical used to
treat acute lymphoblastic leukemia (ALL), the enzyme commonly used in the therapy is from bacteria, these
have shown good activity but cause many severe immune reactions in patients. The search for L-
asparaginase derived and expressed in eukaryotic organisms opens a possibility to reduce adverse reactions.
In this study they were evaluated the induction time (24-120 hours) and inducer concentration $(0.25$, 0.5 and
$1.0 \%$ v/v). The data showed that the condition of increased expression of L-asparaginase II from
Saccharomyces cerevisiae after 48 hours' induction with $1.0 \%$ methanol $(\sim 25$ U.g-1). Finally, it is 
recommended to evaluate this production in bioreactor where adequate control of other important variables such as $\mathrm{pH}$ of the culture and the concentration of oxygen in the medium is carried.

Keywords: Pichia pastoris, Saccharomyces cerevisiae, L-asparaginase, Metanol.

\section{Introducción}

Asparaginasa (EC.3.5.1.1) cataliza la desaminación de la asparagina en aspartato y amonio, además es un importante agente quimioterapeutico para el manejo de leucemia linfoblastica aguda (LLA) y otras enfermedades hematopoyéticas como linfomas noHodgkin [1,2]. La actividad antineoplásica fue observada inicialmente en cobayos como el resultado del hidrolisis de las reservas circulantes de Lasparagina, inhibición de la síntesis de proteínas y apoptosis en células leucémicas susceptibles [1]. Los microrganismos son una fuente eficiente y barata de la enzima [3]. La enzima es producida en gran escala por Escherichia coli y Erwinia chrysanthemi [4], ha demostrado buen potencial en el control de la enfermedad, pero reacciones adversas han sido reportadas en los pacientes tratados [5-7]

Los microrganismos eucariotas como levaduras y hongos filamentosos tienen potencial para producir L-asparaginasa [8, 9]. La levadura Saccharmyces cerevisiae presenta dos tipos de L-asparaginasa con diferente localización, intracelular y periplasmática, estas han sido denominadas como L-asparaginasa I y II respectivamente [10]. Aunque las características de estabilidad y $\mathrm{pH}$ optimo que presenta Lasparaginasa II la presentan como una alternativa de agente antitumoral, la baja cantidad de enzima producida en S. cerevisiae [4] sugieren el uso de un sistema eucariotico de expresión para superar estas desventajas.

La levadura metilotrófica Pichia pastoris es distinguida por su alta producción de biomasa y su potencial para producir grandes cantidades de proteínas recombinantes tanto de organismos procariotas y eucariotas [11]. Un número importante de proteínas han sido exitosamente producidas usando $P$. pastoris, este hecho muestra la relevancia de este sistema de expresión [12, 13]. La inducción de la expresión es controlada por el promotor AOX inducible con metanol [14]. El alcohol oxidasa (AOX) es la primera enzima en el metabolismo de metanol y el genoma de $P$. pastoris contiene dos genes de la enzima funcional: 1) AOX1 responsable por $85 \%$ de la actividad de alcohol oxidasa y 2) AOX2, responsable del $15 \%$ restante [15]. Existen tres fenotipos de P. pastoris definidos por la utilización del metanol: a) Metanol Plus (Mut+) tiene intactos los genes AOX1 y AOX2; b) Metanol Slow (Muts) tiene ausencia del gen AOX1 por lo que crece muy lentamente y una baja utilización de metanol es observada y c) Metanol Minus (Mut-) no crecen en metanol porque ambos genes están ausentes $[16,17]$.

Es por esto último que una de las variables que presentan influencia directa en el rendimiento de expresión son la concentración del agente inductor (metanol) y el tiempo de inducción del cultivo. Por lo tanto, el objetivo de nuestro trabajo fue determinar las mejores condiciones en las que el metanol permite obtener la máxima producción de Lasparaginasa de S. cerevisiae.

\section{Materiales y métodos}

\subsection{Curva de crecimiento del pre-inóculo de $P$. pastoris}

El pre-inóculo de P. pastoris fue llevado a cabo em frascos Erlenmeyer con deflectores de $250 \mathrm{~mL}$ conteniendo $50 \mathrm{~mL}$ de medio BMGY (Buffered Medium Glycerol - Yeast Extract) pH 6.0 con 10 g.L1 de glicerol usado como fuente de carbono. El preinóculo fue iniciado a partir de $1 \mathrm{~mL}$ de suspensión de células conservadas a $-80^{\circ} \mathrm{C}$. La densidad célular fue calculada por espectrometría a $600 \mathrm{~nm}$ durante 36 horas. Los ensayos fueron realizados en tres repeticiones.

\subsection{Cultivo de $P$. pastoris y expresión de L-asparaginasa.}

La enzima fue expresada siguiendo el protocolo del Invitrogen Manual [18], el medio de expresión BMMY (Buffered Medium Methanol - Yeast Extract) pH 6.0 fue inoculado con 1 g.L-1 de células de P. pastoris.

Los experimentos fueron llevados a cabo en frascos Erlenmeyer con deflectores de $250 \mathrm{~mL}$ conteniendo $50 \mathrm{~mL}$ de medio BMMY, los frascos fueron cultivados a $20^{\circ} \mathrm{C}$ y $250 \mathrm{rpm}$ en shaker New Brunswick Scientific $\circledast$. Diferentes concentraciones de metanol $(0.25,0.5$ y $1.0 \%)$ y en diferentes tiempos de inducción (24 - 120 horas) fueron estudiadas y las cantidades de biomasa seca 
producida (g.L-1) y la actividad periplasmática (U.g-1) fueron medidas en todos los puntos. Todos los ensayos fueron realizados tres veces.

\subsection{Cuantificación de la actividad L- asparaginasa}

La actividad L-asparaginasa fue medida en la propia suspensión celular en 100mM de tampón Tris- $\mathrm{HCl}$ pH 7.0 por el método basado en la hidroxilaminolisis de asparagina (conversión de asparagina e hidroxilamina en aspartohidroxamato y amonio). La reacción fue medida por incubación de la suspensión con asparagina e hidroxilamina a $37^{\circ} \mathrm{C}$ en agitación durante 30 minutos. La formación de aspartohidroxamato fue cuantificada por reacción con cloruro férrico y leída en espectrofotómetro a $500 \mathrm{~nm}$.

\section{Resultados y discusión}

La construcción de la curva de crecimiento es importante para determinar el intervalo de tiempo donde es alcanzada la fase exponencial de crecimiento. La velocidad de síntesis de proteínas disminuye cuando la velocidad de crecimiento es menor debido a la disminución de la eficiencia ribosomal [19]. Además, para las levaduras el control de la síntesis de proteínas es dependiente de los controles del ciclo celular, en la fase exponencial la síntesis de proteínas se incrementa exponencialmente; una vez que la célula entra en la fase estacionaria la síntesis de proteínas disminuye más del 90\% [20]. En la Fig. 1 se muestra que la fase exponencial, fase de mayor velocidad de crecimiento, es alcanzada entre las 12 y 20 horas de cultivo.

P. pastoris tiene la capacidad de utilizar el metanol tanto como fuente de carbono, obteniendo energía del metabolismo, como inductor de la expresión de las proteínas heterólogas [21]. Sin embargo, en algunos casos bajas concentraciones de metanol consiguieron inducir mejor la expresión de proteínas heterólogas [22]. Por lo tanto, paralela a la producción de la proteína recombinante, se producirá un incremento de la biomasa presente en el cultivo. En la Fig. 2 observamos que la mayor producción de biomasa fue alcanzada cuando el cultivo fue inducido con $1 \%$ de metanol, menores cantidades de biomasa fueron obtenidos con 0.25 y $0.5 \%$ de metanol. Este hecho es debido a la relación directa que guarda la cantidad de biomasa producida con la cantidad de la fuente de carbono presente en el medio. También observamos que después de las 96 horas de inducción, la producción de biomasa se reduce porque el cultivo está cerca de alcanzar la fase estacionaria de crecimiento.

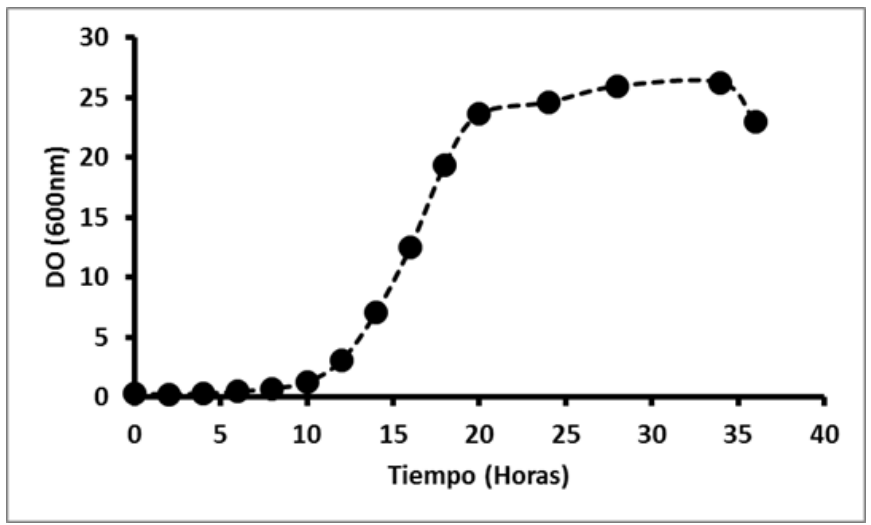

Figura 1. Curva de crecimiento del pre-inóculo de $P$. pastoris cultivada a $30^{\circ} \mathrm{C}, 250 \mathrm{rpm}$ en medio BMGY (pH 6.0).

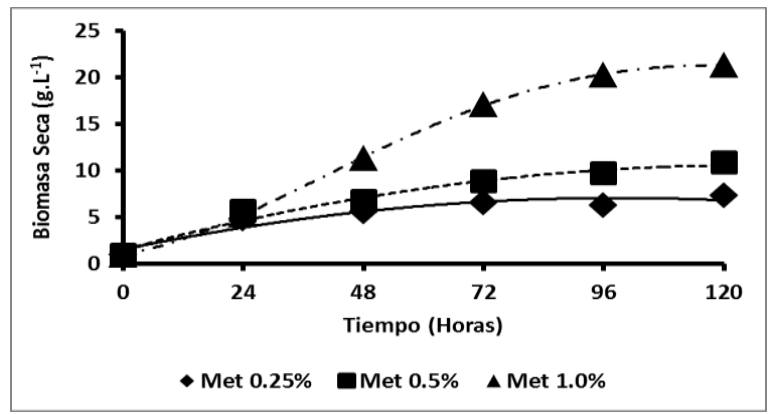

Figura 2. Curva de producción de biomasa en diferentes concentraciones de metanol.

Por su parte, la expresión de L-asparaginasa también fue mayor cuando hubo mayores concentraciones de metanol en el medio. En la Fig. 3 observamos que la actividad periplasmática es

mayor a $1.0 \%$ de metanol, y curva de producción guarda estrecha relación con la curva de producción de biomasa, adicionalmente fue observado un retraso en la producción de Lasparaginasa después de las 48 horas de inducción.

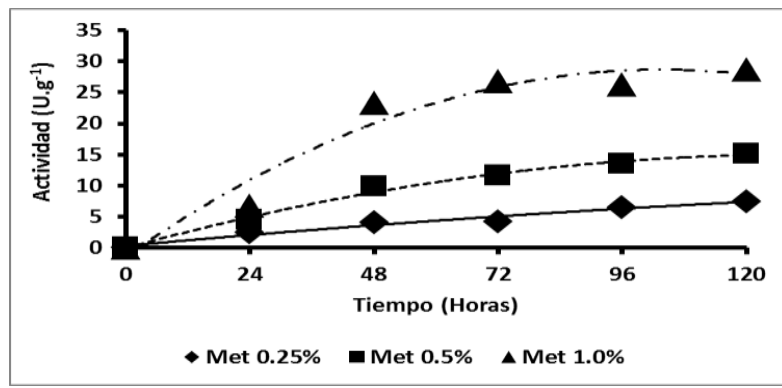


Figura 3. Curva de producción de L-asparaginasa en diferentes concentraciones de metanol.

La producción de L-asparaginasa en las tres concentraciones de metanol después de 48 horas de inducción fue comparada. La Fig. 4 muestra que la cantidad de enzima obtenida a $1 \%$ de metanol fue aproximadamente 6 y 2.5 veces mayor que la cantidad de enzima obtenida con 0.25 y $0.5 \%$ de metanol respectivamente.

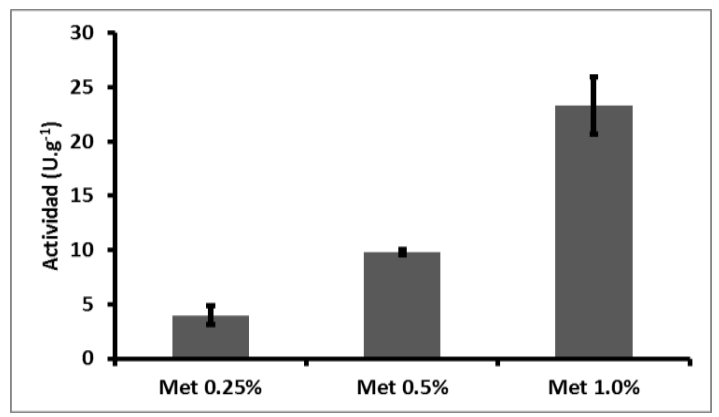

Figure 4. Comparación de la actividad de Lasparaginasa producida en las diferentes concentraciones de metanol después de 48 horas de inducción.

Para finalizar es necesario mencionar que la producción puede ser mejorada aún más a partir de uso de biorreactor donde se lleve un adecuando de otras variables importantes como el control del $\mathrm{pH}$ durante todo el tiempo de cultivo y la cantidad de oxígeno presente en el medio de cultivo que es un sustrato importante en el metabolismo del metanol.

\section{Conclusiones}

La concentración de metanol en el rango $0.25-1 \%$ usado en la inducción guarda relación directa con las cantidades de biomasa y L-asparaginasa producidas. La mejor condición para producción de L-asparaginasa II de $\mathrm{S}$. cerevisiae usando $\mathrm{P}$. pastoris fue de 48 horas de inducción con 1\% de metanol.

\section{Agradecimientos}

Laboratorio de Biotecnología FarmacéuticaFacultad de Ciencias Farmacéuticas de Sao Paulo.

\section{Referencias}

[1] J.L. Shrivastava, A. Arif Khan, M. Khurshid, M. Abul Kalamb. Crit Rev Oncol Hemat. 5 (2015) pp 75-80.

[2] H.J. Müller, J. Boos. Crit Rev Oncol Hemat.
28 (1998) pp 97-113.

[3] U.K. Narta, S.S. Kanwar, W. Azmi. Crit Rev Oncol Hemat. 61 (2007) pp 208221.

[4] M. Ferrara, N. Severino, J. Mansure. Enzyme Microb Technol. 39 (2006) pp 1457-1463.

[5] J. Kurtzberg, B. Asselin, M. Bernstein, G.R. Buchanan. J Pediatr Hematol Oncol. 33 (2011) pp 610-616.

[6] D. Killander, A. Dohlwitz, L. Engstedt. Cancer. 37 (1976) pp 220-228.

[7] T. Onhuma, J.F. Holland, A. Freeman, L.F. Sinks. Cancer Research. 30 (1970) pp 2297-2305.

[8] J.M. Wiame, M. Grenson, N.H. Arst. Adv Microbiol Physiol. 26 (1985) pp 188.

[9] I.O. Pinheiro, J.M. Araujo, E.C. Ximenes, J.C. Pinto. Biomaterial and Diagnostic BD. 6 (2001) pp 243-244.

[10] P.C. Dunlop, G.M. Meyer, D. Ban, R.J. Roon. J Bacteriol 143 (1980) pp 422-6.

[11] J.L. Cereghino, J.M. Cregg. FEMS Microbiol Rev. 24 (2000) pp 45-66.

[12] M. Ahmad, M. Hirz, H. Pichler, H. Schwab. Appl Microbiol Biotechnol. 98 (2014) pp 5301-17.

[13] P. Calik, O. Ata, H. Gunes, A. Massahi, E. Boy, A. Keskin. Biochemical Engineering Journal. 95 (2015) pp 20- 36.

[14] M. Maurer, M. Kuehleitner, B. Gasser, D. Mattanovich. Microb Cell Fact. 5 (2006) pp 37-47.

[15] F. Valero. Protein Eng Technol Appl. 33 (2013) pp 45-48

[16] S. Macauley, M.L. Fazenda, B. McNeil, L.M. Harvey. Yeast, 22 (2005) pp 249- 270.

[17] M.M. Guama, G.J. Lesnicki, B.M. Tam, J. Robinson, C.Z. Radziminski. Biotech Bioeng, 56 (1997) pp 279-286.

[18] INVITROGEN. A Manual of Methods for Expression of Recombinant Proteins in $\mathrm{P}$. pastoris. Cat. No. K171001 (1996).

[19] A.H. Rose, J.S. Harrison. The Yeast, 3 (1989) pp 140-142.

[20] S.G. Elliot, C.S. McLaughlin. Progress in Nucleic Acid Research and Molecular Biology, 8 (1983) pp 87-91.

[21] J.M. Cregg, T.S. Vedvick, and W.C. Raschke. Bio/Technology, 11 (1993) pp 905-910.

[22] Y. Katakura, W. Zhang, G. Zhuang, T. Omasa. Journal of Fermentation and Bioengineering 86 (1998) pp 482-487. 